\title{
Criminologie
}

\section{Agression sexuelle et violence dans les relations amoureuses}

Le rôle médiateur du stress post-traumatique

\section{Sexual abuse and violence in dating relationships}

The mediating role of post-traumatic stress symptoms

\section{Agresión sexual y violencia en las relaciones amorosas} El rol mediador del estrés post-traumático

\section{Martine Hébert, Marie-Ève Daspe, Martin Blais, Francine Lavoie et Mireille Guerrier}

Volume 50, numéro 1, printemps 2017

L'agression sexuelle commise sur des mineurs : les victimes, les auteurs

URI : https://id.erudit.org/iderudit/1039800ar

DOI : https://doi.org/10.7202/1039800ar

Aller au sommaire du numéro

Éditeur(s)

Les Presses de l’Université de Montréal

ISSN

0316-0041 (imprimé)

1492-1367 (numérique)

Découvrir la revue

Citer cet article

Hébert, M., Daspe, M.-È., Blais, M., Lavoie, F. \& Guerrier, M. (2017). Agression sexuelle et violence dans les relations amoureuses : le rôle médiateur du stress post-traumatique. Criminologie, 50(1), 157-179.

https://doi.org/10.7202/1039800ar

\section{Résumé de l'article}

Cette étude vise à documenter le rôle médiateur des symptômes de stress post-traumatique (SSPT) dans le lien entre l'agression sexuelle et différentes formes de victimisation vécues dans le contexte de relations amoureuses. L'étude se fonde sur l'Enquête sur les parcours amoureux des jeunes (PAJ), réalisée au sein de 34 écoles secondaires du Québec. Les jeunes ont rempli un questionnaire portant sur leurs expériences de victimisation, y compris l'agression sexuelle vécue pendant l'enfance et la violence psychologique, physique et sexuelle vécue au sein de leurs relations amoureuses dans les 12 derniers mois. Les SSPT ont été évalués à l'aide du UCLA PTSD Index. Sur un total de 8194 adolescents, $15 \%$ des filles et $4 \%$ des garçons ont rapporté avoir vécu une agression sexuelle. Les résultats des analyses acheminatoires semblent indiquer que le fait d'avoir été victime d'agression sexuelle est associé à davantage de SSPT et qu'en retour, les SSPT sont positivement associés à la violence psychologique, physique et sexuelle subie de la part d'un partenaire amoureux. Cette étude comporte des implications pratiques pour la prévention et l'intervention sur le plan de la violence dans les relations amoureuses chez les victimes d'agression sexuelle. 


\title{
Agression sexuelle et violence dans les relations amoureuses
}

\section{Le rôle médiateur du stress post-traumatique ${ }^{1}$}

\author{
Martine Hébert ${ }^{2}$ \\ Professeure \\ Département de sexologie, Université du Québec à Montréal \\ hebert.m@uqam.ca \\ Marie-Ève Daspe \\ Stagiaire postdoctorale \\ Département de sexologie, Université du Québec à Montréal \\ daspe.marie-eve@courrier.uqam.ca \\ Martin Blais \\ Professeur \\ Département de sexologie, Université du Québec à Montréal \\ blais.martin@uqam.ca \\ Francine Lavoie \\ Professeure \\ École de psychologie, Université Laval \\ francine.lavoie@psy.ulaval.ca \\ Mireille Guerrier \\ Analyste \\ Département de sexologie, Université du Québec à Montréal \\ guerrier.mireille@uqam.ca
}

RÉSUMÉ - Cette étude vise à documenter le rôle médiateur des symptômes de stress post-traumatique (SSPT) dans le lien entre l'agression sexuelle et différentes formes de victimisation vécues dans le contexte de relations amoureuses. L'étude se fonde sur l'Enquête sur les parcours amoureux des jeunes (PAJ), réalisée au sein de 34 écoles

1. Cette recherche a été réalisée grâce à une subvention des Instituts de recherche en santé du Canada (\#103944) octroyée à Martine Hébert. Nous tenons à remercier les adolescentes et les adolescents ayant participé à ce projet ainsi que tous les membres des écoles impliquées. Nous tenons également à remercier Catherine Moreau, coordonnatrice du projet Parcours amoureux des jeunes (PAJ).

2. Département de sexologie, Université du Québec à Montréal, C.P. 8888, succ. Centre-ville, Montréal (Québec), Canada, H3C 3P8. 
secondaires du Québec. Les jeunes ont rempli un questionnaire portant sur leurs expériences de victimisation, y compris l'agression sexuelle vécue pendant l'enfance et la violence psychologique, physique et sexuelle vécue au sein de leurs relations amoureuses dans les 12 derniers mois. Les SSPT ont été évalués à l'aide du UCLA PTSD Index. Sur un total de 8194 adolescents, 15\% des filles et 4\% des garçons ont rapporté avoir vécu une agression sexuelle. Les résultats des analyses acheminatoires semblent indiquer que le fait d'avoir été victime d'agression sexuelle est associé à davantage de SSPT et qu'en retour, les SSPT sont positivement associés à la violence psychologique, physique et sexuelle subie de la part d'un partenaire amoureux. Cette étude comporte des implications pratiques pour la prévention et l'intervention sur le plan de la violence dans les relations amoureuses chez les victimes d'agression sexuelle.

MOTS CLÉS - Agression sexuelle, violence dans les relations amoureuses, stress posttraumatique, médiation, prévention.

\section{Introduction}

Les données de prévalence québécoises estiment que 22,1\% des femmes et 9,7\% des hommes rapportent avoir été victimes d'agression sexuelle avant l'âge de 18 ans et qu'une proportion importante des victimes n'ont jamais dévoilé l'événement (Hébert, Tourigny, Cyr, McDuff et Joly, 2009). Malgré l'effervescence de la recherche scientifique dédiée à ce phénomène, les conséquences de l'agression sexuelle sur les parcours amoureux des victimes, particulièrement à l'adolescence, demeurent peu documentées. L'adolescence est pourtant une période particulièrement déterminante pour le développement relationnel, alors que l'individu fait l'expérience de ses premières relations intimes et amoureuses et se bute à plusieurs tâches développementales, dont le développement de négociation de conflits en contexte romantique.

Parmi les impacts délétères de l'agression sexuelle sur le fonctionnement relationnel, on retrouve notamment le risque de revictimisation dans les relations amoureuses. Chez les populations adultes, un bon nombre d'études ont souligné le risque accru de subir de la violence psychologique, physique ou sexuelle de la part d'un partenaire chez les victimes d'agression sexuelle dans l'enfance (Coid et al., 2001; Daigneault, Hébert et McDuff, 2009; Desai, Arias, Thompson et Basile, 2002; DiLillo, Giuffre, Tremblay et Peterson, 2001; Whitfield, Anda, Dube et Felitti, 2003). Chez les adolescents, les rares études ayant examiné cette association montrent également que les jeunes présentant un historique d'agression sexuelle sont nombreux à rapporter subir de la violence de la part d'un partenaire amoureux (Cyr, McDuff et Wright, 2006; Hébert, Brabant et Daigneault, 2013 ; Manseau, Fernet, Hébert, 
Collin-Vézina et Blais, 2008) et qu'ils sont significativement plus à risque que les jeunes n'ayant pas vécu une agression sexuelle (Hébert, Lavoie, Vitaro, McDuff et Tremblay, 2008; Tourigny, Lavoie, Vézina et Pelletier, 2006). Peu d'études ont cependant tenté de répliquer l'association entre l'agression sexuelle et la violence subie en contexte amoureux auprès d'échantillons représentatifs d'adolescents et d'adolescentes. De plus, les mécanismes explicatifs de cette association demeurent peu étayés. Or, le développement de programmes de prévention pour cette population vulnérable requiert une meilleure connaissance des facteurs qui contribuent à la revictimisation au sein des premières relations amoureuses.

Mécanismes explicatifs de la relation entre l'agression sexuelle et la violence subie dans les relations amoureuses

Malgré la rareté des données empiriques, certains modèles conceptuels offrent des pistes afin de mieux comprendre les mécanismes en jeu dans la relation entre l'agression sexuelle et la revictimisation dans les relations amoureuses adolescentes (Hébert, Daigneault et Van Camp, 2012). Parmi ceux-ci, le modèle des dynamiques traumatogènes de Finkelhor et Browne (1985) semble indiquer qu'un sentiment d'impuissance est souvent ressenti chez les victimes d'agression sexuelle et que celui-ci est susceptible d'inhiber les comportements d'autoprotection lorsque l'individu doit à nouveau faire face à des situations à risque de victimisation (Banyard, Arnold et Smith, 2000). De la même manière, l'expérience d'une agression sexuelle est susceptible de diminuer les capacités de régulation émotionnelle et, subséquemment, de rendre l'individu moins enclin à détecter les comportements violents à son endroit ou à mettre fin à une relation violente (Banyard et al., 2000). Enfin, l'agression sexuelle dans l'enfance est associée à la fois à un plus jeune âge lors des premières relations sexuelles et à un nombre plus élevé de partenaires, constituant tous deux des facteurs de risque de subir de la violence dans les relations amoureuses (Vézina et Hébert, 2007). Bien que différents modèles aient été proposés pour expliquer la revictimisation, peu de modèles ont été validés empiriquement à ce jour. Parmi les différents mécanismes proposés, le rôle des symptômes de stress post-traumatique comme variables intermédiaires pouvant influer sur le risque de revictimisation chez les personnes ayant vécu une agression sexuelle a retenu l'attention de plusieurs chercheurs. Les travaux pertinents seront décrits dans la prochaine section. 


\section{Symptômes de stress post-traumatique}

Une piste particulièrement pertinente afin de comprendre les impacts de l'agression sexuelle sur les risques de victimisation à l'adolescence implique l'état de stress post-traumatique. Les manifestions cliniques de ce trouble comprennent des symptômes d'intrusion, d'évitement des stimuli associés à l'événement traumatisant et d'hyperéveil. Ainsi, parmi l'éventail des répercussions associées à l'agression sexuelle vécue pendant l'enfance, les SSPT sont parmi les plus fréquemment retrouvés chez les victimes d'agression sexuelle (Oddone Paolucci, Genuis et Violato, 2001), avec $57 \%$ des adolescents présentant un historique d'agression sexuelle qui souffrent de SSPT atteignant le seuil clinique (Nooner et al., 2012). L'adolescence constituerait une période critique pour le développement des SSPT. En effet, les jeunes sont exposés à plus d'événements traumatiques que les adultes et présentent des taux de SSPT plus élevés que ces derniers (Nooner et al., 2012). Sur le plan des différences liées au genre, si certaines observations indiquent que les filles présentent des taux d'état de stress post-traumatique deux fois plus élevés que les garçons (Kilpatrick et al., 2003 ; Nooner et al., 2012), d'autres semblent indiquer que les garçons et les filles présentent des taux équivalents (Maikovich, Koenen et Jaffee, 2009).

Différentes explications sont proposées pour expliquer le lien entre les SSPT et la victimisation ultérieure. La relation entre les SSPT et la victimisation au sein des relations amoureuses s'expliquerait notamment par le fait que la nature des symptômes taxerait la capacité de l'individu à cerner les situations à risque et à déployer des stratégies de protection relativement à ces situations (Arata, 2000; Chu, 1992; Orcutt, Erickson et Wolfe, 2002). Un effet médiateur des SSPT a ainsi été observé dans la relation entre l'agression sexuelle dans l'enfance et la revictimisation sexuelle à l'âge adulte (Arata, 2000; Messman-Moore, Brown et Koelsch, 2005). Un effet médiateur réfère ici à une variable intermédiaire qui représente le mécanisme par lequel l'agression sexuelle est associée au risque de revictimisation. Dans une étude ayant examiné plus spécifiquement la contribution des différentes dimensions des SSPT (intrusion, évitement et hyperéveil), seuls les symptômes d'hyperéveil joueraient un rôle médiateur dans la relation entre l'agression sexuelle dans l'enfance et la victimisation sexuelle à l'âge adulte (Risser, Hetzel-Riggin, Thomsen et McCanne, 2006). Ainsi, des niveaux d'hyperéveil chroniques pourraient être associés à une plus faible capacité à faire la discrimination entre fausses alarmes et signaux de danger réels, condui- 
sant éventuellement l'individu à négliger les signes de menaces et l'exposant ainsi à un plus grand risque de revictimisation. Dans un échantillon de 349 étudiantes universitaires, Sandberg, Matorin et Lynn (1999) n'ont cependant observé aucun effet médiateur des SSPT dans la relation entre l'agression sexuelle dans l'enfance ou à l'adolescence et la revictimisation, ceux-ci n'ayant pas permis de prédire l'occurrence de victimisation à l'âge adulte.

Si les études précédemment recensées ont toutes exploré la revictimisation sexuelle sans égard à l'identité de l'agresseur, d'autres auteurs se sont intéressés plus spécifiquement à la violence perpétrée par un partenaire amoureux. Au sein d'un échantillon de femmes adultes, Messing, La Flair, Cavanaugh, Kanga et Campbell (2012) ont observé un effet médiateur des SSPT sur la relation entre l'agression sexuelle dans l'enfance et la violence psychologique et sexuelle de la part d'un partenaire amoureux, et ce, en contrôlant les effets des autres formes de mauvais traitements dans l'enfance. Les auteurs n'ont toutefois pas observé de relation significative entre les SSPT et la violence physique subie. Dans un échantillon de femmes en traitement à la méthadone, Engstrom, El-Bassel, Go et Gilbert (2008) ont également observé un effet médiateur de la relation entre les SSPT et la violence psychologique, physique ou sexuelle subie de la part d'un partenaire.

Jusqu'à maintenant, les études portant sur la contribution des SSPT à la revictimisation ont été réalisées presque exclusivement avec des échantillons de femmes adultes. À notre connaissance, aucune étude n'a examiné le rôle médiateur des SSPT dans la relation entre l'agression sexuelle et la violence subie dans les relations amoureuses au sein de populations adolescentes. De plus, la présence de différences liées au genre au sein de ces associations n'a jamais été investiguée puisque les études sont généralement réalisées exclusivement auprès de femmes victimes d'agression sexuelle. Enfin, à l'exception de l'étude de Messing et al. (2012), ayant exploré l'impact de l'agression sexuelle et des SSPT sur les différentes formes de violence dans les relations amoureuses, les conséquences sur la violence psychologique et physique sont très peu documentées. Pourtant, il est possible que la contribution des SSPT à la relation entre l'agression sexuelle et la revictimisation dans les relations amoureuses diffère selon le type de violence subie. Par exemple, certaines données empiriques semblent indiquer que la relation entre les SSPT et la violence subie de la part d'un partenaire amoureux est plus forte pour la violence psychologique que pour les autres formes 
de violence (voir entre autres Taft, Murphy, King, Dedeyn et Musser, 2005).

Dans ce contexte, la présente étude vise à examiner l'effet médiateur des SSPT dans la relation entre l'agression sexuelle et la revictimisation psychologique, physique et sexuelle, spécifiquement au sein des relations amoureuses. Elle constitue la première tentative d'investiguer le rôle des SSPT dans la victimisation au sein d'une population adolescente. L'utilisation d'un échantillon représentatif de la population québécoise permet de documenter le rôle des SSPT tant chez les filles que chez les garçons, et de vérifier l'impact du genre sur les relations examinées.

\section{Méthodologie}

\section{Participants et procédures}

Les données de la présente étude sont tirées de l'enquête longitudinale sur les parcours amoureux des jeunes (PAJ). PAJ est une étude dont l'objectif principal vise à documenter la violence dans les relations amoureuses chez les jeunes de 14 à 18 ans, à décrire leurs expériences de victimisation et à en définir les corrélats. Au premier temps de mesure, les participants ont été recrutés selon un plan d'échantillonnage stratifié en grappes à un degré d'écoles secondaires du Québec. Les écoles ont été sélectionnées aléatoirement à partir d'une liste d'écoles admissibles du ministère de l'Éducation, du Loisir et du Sport. Ces écoles ont été regroupées en huit strates en tenant compte de la région géographique, du réseau d'enseignement (privé ou public), de la langue d'enseignement (français ou anglais) et de l'indice de défavorisation, afin d'obtenir un échantillon représentatif des adolescents de $3^{\mathrm{e}}, 4^{\mathrm{e}}$ et $5^{\mathrm{e}}$ secondaire. Le comité d'éthique de l'Université du Québec à Montréal a approuvé ce projet et les participants volontaires ont signé un formulaire de consentement stipulant qu'ils pouvaient suspendre leur participation à n'importe quel moment sans avoir à donner d'explication.

Au total, 34 écoles ont participé à l'enquête. Afin d'examiner le taux de réponse par classe et le taux de réponse global des élèves, un rapport entre le nombre d'élèves ayant accepté de participer (les élèves pour lesquels un consentement était obtenu) et le nombre d'élèves à qui on a proposé de participer par classe et sur l'ensemble des participants a été calculé. Un taux de réponse de $100 \%$ a été obtenu pour la majorité 
des classes (320 sur un total de 329 classes); pour les classes restantes $(9 / 329)$, le taux de réponse varie de $90 \%$ à $98 \%$. Le taux global de réponse s'élève à $99 \%$. L'échantillon total est constitué de 8194 jeunes. Une pondération a été utilisée afin de tenir compte du biais d'échantillonnage. Pour un participant dans un niveau scolaire donné, le poids attribué est défini comme l'inverse de la probabilité de sélection du participant selon ce niveau scolaire dans la strate de l'échantillon, multiplié par la probabilité de sélection du participant selon ce même niveau scolaire dans la strate dans la population. La taille de l'échantillon pondéré au Temps 1 est de 6531 jeunes.

Cet échantillon est composé de 3776 (57,8\%) filles et de 2755 $(42,2 \%)$ garçons. La moyenne d'âge des participants était de 15,35 $(E S=0,10)$ chez les filles et de 15,36 $(E S=0,12)$ chez les garçons. Sur le plan du degré de scolarité, $37,0 \%$ des participants $(n=2419)$ étaient inscrits en $3^{\mathrm{e}}$ secondaire, $31,4 \%(n=2050)$ étaient en $4^{\mathrm{e}}$ secondaire et $31,6 \%(n=2062)$ étaient en $5^{\mathrm{e}}$ secondaire. Au sein de l'échantillon, $75,4 \%(n=4908)$ des jeunes étaient francophones, 3,6\% $(n=236)$ étaient anglophones et $21,0 \%(n=1366)$ avaient une autre langue maternelle ou une combinaison de langues maternelles. En ce qui a trait à la structure familiale, $63,2 \%(n=4118)$ des jeunes vivaient avec leurs deux parents, $12,7 \%(n=829)$ vivaient en garde partagée, $21,9 \%$ $(n=1428)$ vivaient en famille monoparentale et $2,2 \%(n=140)$ ont rapporté une structure familiale autre.

\section{Mesures}

\section{Violence dans les relations amoureuses}

Les participants ont rempli une échelle autorapportée adaptée du Conflict in Adolescent Dating Relationships Inventory (CADRI; Wolfe et al., 2001) permettant de décrire la violence survenue au sein d'une relation amoureuse dans les 12 derniers mois. Dans le cadre de la présente étude, seule la violence subie de la part du partenaire a été considérée. Cette version courte du CADRI évalue entre autres la violence psychologique et la violence physique à l'aide de trois énoncés par sous-échelle. Des exemples d'items réfèrent à: «Au cours des 12 derniers mois, à quelle fréquence ton chum ou ta blonde a fait ces gestes envers toi? ... Te suivre pour savoir où et avec qui tu es?» ou «Donner une gifle ou tirer les cheveux?» L'instrument original présente des qualités psychométriques adéquates avec des coefficients $\alpha$ de 0,82 
$(0,81$ pour les garçons et 0,82 pour les filles) et $0,82(0,86$ pour les garçons et 0,80 pour les filles) pour les échelles de violence psychologique et physique respectivement. Au sein de la présente étude, les coefficients sont de 0,56 et 0,71.

La violence sexuelle a été mesurée à l'aide de neuf énoncés adaptés d'une version révisée du Sexual Experiences Survey (SES; Koss et al., 2007; Koss et Gidycz, 1985; Koss et Oros, 1982). Dans le cadre de la présente étude, cette adaptation du questionnaire a permis d'évaluer la violence sexuelle perpétrée spécifiquement par un partenaire amoureux. Un exemple d'item réfère à: "Au cours des 12 derniers mois, à quelle fréquence ton chum ou ta blonde a fait ces gestes envers toi? ... Essayer d'avoir une relation sexuelle alors que tu ne voulais pas? ... En utilisant un certain degré de force physique pour t'obliger?» Les choix de réponse aux énoncés sont basés sur une échelle de type Likert en 4 points $(0=$ jamais, $1=1$ ou 2 fois, $2=3$ à 5 fois, $3=6$ fois et plus $)$. La version originale du SES (Koss et Gidycz, 1985) présente des qualités psychométriques adéquates avec un coefficient $\alpha$ de 0,74 pour les femmes et de 0,89 pour les garçons. Dans la présente étude, le coefficient pour l'échantillon total est de 0,89 .

À l'aide du CADRI et du SES, un score total pour chacune des formes de violence a été dérivé selon la moyenne des items de l'échelle en question.

\section{Agression sexuelle}

L'expérience d'agression sexuelle a été mesurée à l'aide de deux questions adaptées de Finkelhor, Hotaling, Lewis et Smith (1990), comme utilisé dans le contexte d'un sondage auprès de la population adulte québécoise (Tourigny et al., 2008). Un premier énoncé réfère à une agression impliquant des attouchements non désirés: «As-tu déjà été touché sexuellement alors que tu ne le voulais pas ou été contraint ou obligé par manipulation, chantage ou utilisation de la force de toucher sexuellement?» Un deuxième énoncé visait à évaluer les situations d'agression impliquant une relation sexuelle avec pénétration: «À l'exception des attouchements sexuels mentionnés, as-tu déjà été contraint ou obligé par manipulation, chantage ou utilisation de la force d'avoir une relation sexuelle avec pénétration (orale, vaginale ou anale)? » Les participants ont répondu par Oui ou Non à chacun de ces items. Un score dichotomique d'agression sexuelle a été créé en attribuant un score de 1 aux participants ayant répondu par l'affir- 
mative à au moins un item et un score de 0 aux participants ayant répondu par la négative aux deux items. Les participants ayant endossé l'un ou l'autre des énoncés étaient invités à préciser qui était la personne impliquée (membre de la famille immédiate ou élargie, personne en situation d'autorité ou personne inconnue). Les énoncés ont été formulés de façon à exclure les situations de violence vécue en contexte amoureux qui sont évaluées dans la section sur la violence dans les relations.

\section{Symptômes de stress post-traumatique}

L'Abbreviated University of California at Los Angeles PTSD Reaction Index (Steinberg, Brymer, Decker et Pynoos, 2004) a été utilisé pour mesurer les SSPT. Ce questionnaire a été élaboré aux fins de dépistage et de détermination des besoins aux fins d'évaluation et de référence clinique (Steinberg et al., 2004). Les neuf énoncés de ce questionnaire sont mesurés selon une échelle de Likert en cinq points allant de 0 (jamais) à 4 (toujours). La cote globale est obtenue en additionnant les réponses et varie de 0 à 36 . Les indices de consistance interne de la version originale sont adéquats de même que la sensibilité pour déceler les SSPT (Cohen, 2010). Au sein de la présente étude, le coefficient $\alpha$ est de 0,88 .

\section{Variables sociodémographiques}

Un questionnaire sociodémographique a recueilli les informations relatives au genre, à l'âge, au degré de scolarité, à la langue parlée à la maison (français, anglais, autre) et à la structure familiale (si le jeune vit avec ses deux parents, un seul de ses parents ou dans une autre structure familiale).

\section{Analyses statistiques}

Les analyses statistiques ont été conduites à l'aide du logiciel Stata (StataCorp., 2011). Dans un premier temps, des analyses descriptives ont été réalisées afin d'examiner les corrélations entre les variables à l'étude. La présence de différences liées au genre a également été vérifiée. Pour la variable dichotomique d'agression sexuelle, un test du chi carré avec correction de deuxième ordre de Rao et Scott (Rao et Scott, 1981, 1984) a été utilisé afin de corriger en fonction du devis complexe 
de l'enquête. Le chi carré est ainsi transformé en une statistique $F$ (Fisher). Des tests-t pour échantillons indépendants ont été réalisés pour examiner les différences de genre dans les scores de SSPT et des différents types de violence. De la même manière, la présence de différences significatives entre les victimes d'agression sexuelle et les non-victimes d'agression sexuelle dans les scores de SSPT et de violence dans les relations amoureuses a été examinée.

Dans un second temps, le rôle médiateur des SSPT dans la relation entre l'agression sexuelle et la violence dans les relations amoureuses a été examiné à l'aide d'analyses acheminatoires. L'effet indirect de l'agression sexuelle sur la violence via le SSPT ainsi que le pourcentage de l'effet total représenté par cet effet indirect ont été estimés. Au sein du modèle, l'effet modérateur du genre dans la relation entre l'agression sexuelle et le SSPT ainsi que dans la relation entre l'agression sexuelle et la violence dans les relations amoureuses a été exploré (Figure 1). Cet effet modérateur a été estimé en créant un terme d'interaction entre l'agression sexuelle et le genre. Ce terme d'interaction a été ensuite introduit dans le modèle de médiation. Enfin, puisque certaines observations empiriques indiquent une augmentation de la violence entre le début et le milieu de l'adolescence, suivie d'une diminution vers la fin de l'adolescence (par ex., Brooks-Russell, Foshee et Ennett, 2013), l'effet curvilinéaire (en forme de U) de l'âge a été analysé en introduisant l'âge des participants et l'âge au carré dans les modèles. Un modèle de médiation distinct a été estimé pour chacune des trois formes de violence considérées (psychologique, physique et sexuelle).

F I G URE 1

Modèle conceptuel: agression sexuelle, symptômes de stress post-traumatique et violence dans les relations amoureuses

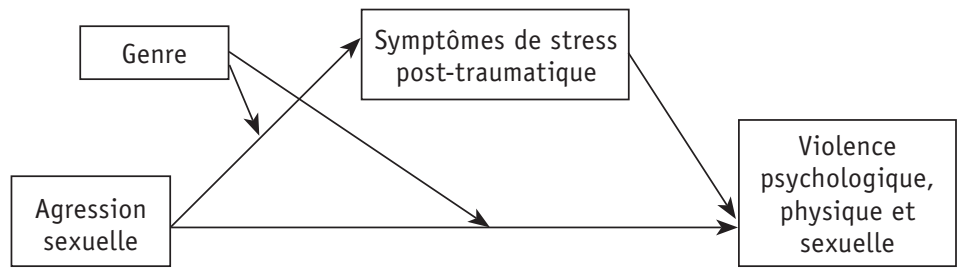




\section{Résultats}

\section{Analyses descriptives}

Les statistiques descriptives pour les différentes variables à l'étude sont présentées dans le Tableau 1. Les résultats d'analyses de corrélation indiquent que l'agression sexuelle, les SSPT et les différents types de violence sont tous significativement et positivement corrélés. Le genre féminin est également positivement associé à l'agression sexuelle, aux SSPT et à la violence psychologique et sexuelle. Aucune corrélation entre le genre et la violence physique n'est observée. L'âge des participants est quant à lui positivement corrélé à l'agression sexuelle ainsi qu'aux trois formes de violence, mais n'est pas significativement associé aux SSPT.

TA B LEA U 1

Statistiques descriptives et coefficients de corrélation pour les variables à l'étude

\begin{tabular}{|l|c|c|c|c|c|c|c|}
\hline Variables & 1 & 2 & 3 & 4 & 5 & 6 & 7 \\
\hline 1. AS & - & & & & & & \\
\hline 2. SSPT & $0,25^{* * *}$ & - & & & & & \\
\hline 3. VRA psychologique & $0,15^{* * *}$ & $0,20^{* * *}$ & - & & & & \\
\hline 4. VRA physique & $0,11^{* * *}$ & $0,15^{* * *}$ & $0,40^{* * *}$ & - & & & \\
\hline 5. VRA sexuelle & $0,16^{* * *}$ & $0,12^{* *}$ & $0,34^{* * *}$ & $0,39^{* * *}$ & - & & \\
\hline 6. Genre féminin & $0,18^{* * *}$ & $0,27^{* * *}$ & $0,10^{* * *}$ & 0,03 & $0,12^{* * *}$ & - & \\
\hline 7. Âge & $0,07^{* * *}$ & 0,03 & $0,14^{* * *}$ & $0,08^{* *}$ & $0,08^{* *}$ & $-0,01$ & - \\
\hline
\end{tabular}

Notes: $A S=$ agression sexuelle; $S S P T=$ symptômes de stress post-traumatique; $V R A=$ violence dans les relations amoureuses. ${ }^{* * *} p<0,001 .{ }^{* *} p<0,01$.

Au sein de l'échantillon, 10,3\% $(n=659)$ des participants rapportent avoir été victimes d'agression sexuelle, avec une proportion significativement plus élevée de filles $(14,9 \%, n=553)$ que de garçons $(3,9 \%$, $n=106), F(1,26)=108,32, p<0,001$. Les résultats indiquent également des niveaux significativement plus élevés de SSPT chez les filles $(M=10,67, E S=0,21)$ comparativement aux garçons $(M=6,10$, $E S=0,21), t(26)=10,51, p<0,001$. Les filles rapportent aussi avoir vécu davantage de violence psychologique $(0,41$ vs $0,31, t(26)=6,4$, $p<0,001)$, physique $(0,11$ vs $0,09, t(26)=2,12, p=0,04)$ et sexuelle $(0,07$ vs $0,02, t(26)=7,27, p<0,001)$ de la part d'un partenaire amoureux que les garçons. 
Pour les SSPT et les différentes formes de violence dans les relations amoureuses, le Tableau 2 présente les moyennes et erreurs types pour chacune des variables de l'étude ainsi que les résultats des analyses de comparaison des victimes et des non-victimes d'agression sexuelle. Les résultats indiquent que les jeunes rapportant avoir été victimes d'agression sexuelle présentent des scores significativement plus élevés de SSPT et de victimisation dans les relations amoureuses, sans égard à la forme de violence, relativement à leurs pairs qui ne dévoilent pas d'agression sexuelle pendant l'enfance. Les données indiquent par ailleurs que les victimes d'agression sexuelle sont plus nombreuses à présenter des SSPT atteignant le seuil clinique $(27,9 \%, n=173)$, comparativement aux non-victimes $(9,1 \%, n=310), F(1,26)=185,16, p<0,001$.

TABLEA U 2

Différences sur le plan des symptômes de stress post-traumatique et de la violence dans les relations amoureuses entre les victimes d'agression sexuelle et les non-victimes

\begin{tabular}{|l|c|c|c|c|c|c|}
\hline \multirow{2}{*}{ Variables } & \multicolumn{4}{|c|}{ Agression sexuelle } & \multicolumn{2}{|c|}{} \\
\cline { 2 - 7 } & \multicolumn{2}{|c|}{ Non } & \multicolumn{2}{c|}{ Oui } & \multicolumn{2}{c|}{} \\
\cline { 2 - 7 } & $M$ & $E T$ & $M$ & $E T$ & $T$ & $p$ \\
\hline SSPT & 8,09 & 0,19 & 13,77 & 0,36 & 16,45 & $<0,001$ \\
\hline Violence psychologique & 0,34 & 0,01 & 0,55 & 0,02 & 7,34 & $<0,001$ \\
\hline Violence physique & 0,09 & 0,01 & 0,19 & 0,02 & 5,13 & $<0,001$ \\
\hline Violence sexuelle & 0,04 & 0,00 & 0,13 & 0,01 & 7,82 & $<0,001$ \\
\hline
\end{tabular}

Notes: $M=$ moyenne; $E T$ = erreur type; $S S P T$ = symptômes de stress post-traumatique.

\section{Analyses de médiation}

Association entre l'agression sexuelle et les SSPT

Les résultats d'analyses acheminatoires (Tableau 3) semblent indiquer que le fait d'avoir été agressé sexuellement prédit davantage de SSPT. Bien que le fait d'être une femme prédise également des niveaux plus élevés de SSPT, aucun effet modérateur du genre sur la relation entre l'agression sexuelle et les SSPT n'est observé. Les résultats semblent également indiquer une absence de relation linéaire ou curvilinéaire entre l'âge et les SSPT. 


\section{Violence psychologique}

L'analyse du lien direct entre l'agression sexuelle et la violence psychologique subie de la part du partenaire amoureux révèle une association significative avant l'entrée du médiateur au modèle, $b=0,18, p<0,001$, et ce, en contrôlant l'effet de l'âge et du genre du participant. Les analyses acheminatoires incluant les SSPT (Tableau 3) semblent indiquer que ceux-ci prédisent positivement la violence psychologique subie dans le contexte des relations amoureuses. Au sein de ce modèle de médiation, le lien direct entre l'agression sexuelle et la violence psychologique s'avère non significatif, laissant entendre une médiation complète des SSPT. Les résultats semblent également indiquer un lien indirect significatif via les SSPT qui représente 17,2\% de l'effet total de l'agression sexuelle sur la violence psychologique. Aucun effet d'interaction entre l'agression sexuelle et les SSPT n'est observé dans la prédiction de la violence psychologique. Le fait d'être une femme prédit davantage de violence psychologique subie dans les relations amoureuses, sans toutefois que le genre modère le lien entre l'agression sexuelle et la violence psychologique. Enfin, aucun effet lié à l'âge (linéaire ou curvilinéaire) n'a été trouvé significatif pour le modèle de médiation de la violence psychologique au sein des relations amoureuses. Dans l'ensemble, le modèle de médiation permet de prédire $15 \%$ de la variance de la violence psychologique subie au sein des relations amoureuses.

\section{Violence physique}

Avant l'entrée des SSPT au modèle, les analyses semblent indiquer un lien direct significatif entre l'agression sexuelle et la violence physique subie, $b=0,10, p<0,001$, et ce, en contrôlant les effets des covariables. Les résultats des analyses de médiation laissent entendre que les SSPT permettent également de prédire la violence physique (Tableau 3). Après l'entrée du médiateur au modèle, le lien direct allant de l'agression sexuelle à la violence physique n'est plus significatif, semblant indiquer une médiation complète des SSPT. Le lien indirect significatif via les SSPT représente $14,7 \%$ de l'effet total de l'agression sexuelle sur la violence physique. Aucun effet d'interaction entre l'agression sexuelle et les SSPT n'est observé dans la prédiction de la violence physique. En ce qui a trait aux covariables, les résultats indiquent que le genre ne permet ni de prédire la violence physique ni de modérer son association 
TA B LE A U 3

Modèle de médiation de la relation entre l'agression sexuelle et les différentes formes de violence dans les relations amoureuses via les symptômes de stress post-traumatique

\begin{tabular}{|c|c|c|c|c|c|}
\hline & $B$ & ET & $T$ & IC $95 \%$ & $R^{2}$ \\
\hline \multicolumn{5}{|l|}{ SSPT } & 0,12 \\
\hline AS & $0,53^{* * *}$ & 0,11 & 4,58 & {$[0,29-0,76]$} & \\
\hline Genre & $0,46^{* * *}$ & 0,04 & 11,38 & {$[0,38-0,55]$} & \\
\hline AS * Genre & 0,04 & 0,12 & 0,43 & {$[-0,19-0,30]$} & \\
\hline Âge & 0,85 & 0,70 & $-0,58$ & {$[-0,58-2,29]$} & \\
\hline$\hat{A} g \mathrm{e}^{2}$ & $-0,92$ & 0,73 & $-0,58$ & {$[-2,42-0,58]$} & \\
\hline \multicolumn{5}{|c|}{ VRA psychologique } & 0,15 \\
\hline SSPT & $0,07^{* * *}$ & 0,01 & 6,26 & {$[0,05-0,10]$} & \\
\hline AS & 0,18 & 0,10 & 1,87 & {$[-0,02-0,38]$} & \\
\hline Genre & $0,06^{* *}$ & 0,02 & 3,06 & {$[0,02-0,11]$} & \\
\hline AS * Genre & $-0,09$ & 0,13 & $-0,72$ & {$[-0,36-0,17]$} & \\
\hline $\mathrm{AS}$ * SSPT & 0,04 & 0,03 & 1,16 & {$[-0,03-0,11]$} & \\
\hline Âge & $-0,17$ & 0,51 & $-0,34$ & {$[-1,22-0,88]$} & \\
\hline$\hat{\mathrm{Age}^{2}}$ & 0,26 & 0,55 & 0,47 & {$[-0,87-1,38]$} & \\
\hline Effet indirect & $0,04^{* * *}$ & 0,01 & 3,82 & {$[0,02-0,06]$} & \\
\hline \multicolumn{5}{|l|}{ VRA physique } & 0,14 \\
\hline SSPT & $0,04^{* * *}$ & 0,01 & 5,69 & {$[0,03-0,06]$} & \\
\hline AS & 0,14 & 0,08 & 1,64 & {$[-0,04-0,31]$} & \\
\hline Genre & 0,00 & 0,01 & $-0,2$ & {$[0,02-0,19]$} & \\
\hline AS * Genre & $-0,09$ & 0,09 & $-1,04$ & {$[-0,27-0,09]$} & \\
\hline$A S * S S T P$ & 0,01 & 0,02 & 0,58 & {$[-0,03-0,05]$} & \\
\hline Âge & $-0,49$ & 0,34 & $-1,42$ & {$[-1,19-0,22]$} & \\
\hline$\hat{A} g \mathrm{e}^{2}$ & 0,54 & 0,37 & 1,49 & {$[-0,21-1,30]$} & \\
\hline Effet indirect & $0,02^{* * *}$ & 0,01 & 3,07 & {$[0,01-0,04]$} & \\
\hline \multicolumn{5}{|l|}{ VRA sexuelle } & 0,16 \\
\hline SSTP & $0,01^{*}$ & 0,01 & 2,51 & {$[0,002-0,03]$} & \\
\hline AS & 0,05 & 0,03 & 1,56 & {$[-0,02-0,11]$} & \\
\hline Genre & $0,04^{* * *}$ & 0,01 & 4,52 & {$[0,02-0,06]$} & \\
\hline AS * Genre & 0,00 & 0,04 & 0,09 & {$[-0,08-0,09]$} & \\
\hline AS * SSTP & 0,01 & 0,02 & 0,75 & {$[-0,02-0,05]$} & \\
\hline Âge & $-0,67^{*}$ & 0,31 & $-2,19$ & {$[-1,31--0,04]$} & \\
\hline$\hat{\mathrm{Age}^{2}}$ & $0,74^{*}$ & 0,33 & 2,23 & {$[0,06-1,41]$} & \\
\hline Effet indirect & $0,007^{*}$ & 0,003 & 2,15 & {$[0,0003-0,01]$} & \\
\hline
\end{tabular}

Notes: $\mathrm{AS}=$ agression sexuelle; $\mathrm{VRA}=$ violence dans les relations amoureuses; $\mathrm{SSPT}=$ symptômes de stress post-traumatique. ${ }^{* *} p<0,001 .{ }^{* *} p<0,01 .{ }^{*} p<0,05$. 
avec l'agression sexuelle. Aucun effet lié à l'âge des participants (linéaire ou curvilinéaire) n'a été trouvé significatif pour le modèle de médiation de la violence physique subie au sein des relations amoureuses. Le modèle de médiation permet d'expliquer $14 \%$ de la variance de la violence physique dans les relations amoureuses.

\section{Violence sexuelle}

L'examen du lien direct avant l'entrée du médiateur au modèle semble indiquer une association significative entre l'agression sexuelle et la violence sexuelle subie, $b=, 07, p<0,001$, en contrôlant les effets des covariables. Les analyses de médiation révèlent que les SSPT permettent aussi de prédire significativement la violence sexuelle en contexte amoureux. Encore une fois, le lien direct entre l'agression sexuelle et la violence sexuelle n'est plus significatif après l'entrée du médiateur au modèle, laissant entendre une médiation complète via les SSPT. Le lien indirect significatif via les SSPT représente 13,7\% de l'effet total de l'agression sexuelle sur la violence sexuelle. Aucun effet d'interaction entre l'agression sexuelle et les SSPT n'est observé dans la prédiction de la violence sexuelle. Les résultats indiquent également que le fait d'être une femme prédit davantage de violence sexuelle, sans toutefois modérer l'association entre l'agression sexuelle et la violence au sein des relations amoureuses. Finalement, contrairement aux deux modèles précédents, les résultats laissent entendre qu'en plus du lien linéaire négatif entre l'âge et la violence sexuelle subie $(b=-0,67, p=0,038)$, il existe aussi un effet curvilinéaire positif $(b=0,74, p=0,035)$. Cet effet curvilinéaire semble indiquer que la violence sexuelle subie dans le contexte des relations amoureuses tend à diminuer chez les jeunes jusqu'à un certain âge (le point d'inflexion se situant à 15,09 ans), à partir duquel la violence sexuelle subie augmente. Le modèle permet d'expliquer $16 \%$ de la variable de la violence sexuelle au sein des relations amoureuses.

\section{Discussion}

Au sein d'un échantillon représentatif des jeunes Québécois, les résultats de la présente étude indiquent que les adolescentes et adolescents ayant vécu une agression sexuelle présentent davantage de SSPT que leurs pairs n'ayant pas vécu d'agression sexuelle. De plus, les données indiquent qu'une proportion importante des adolescents victimes 
d'agression sexuelle affiche des SSPT atteignant le seuil clinique (28\%) comparativement aux adolescents ne rapportant pas d'historique d'agression sexuelle (9\%). Cette proportion dépasse largement les indices de prévalence trouvés auprès d'échantillons normatifs. À titre d'exemple, à l'aide d'un échantillon représentatif d'adolescents américains, Kilpatrick et al. (2003) rapportent que la prévalence de l'état de stress post-traumatique se situe à $6,3 \%$ chez les filles âgées de 12 à 17 ans et à 3,7\% chez les garçons.

La présente étude visait à examiner les SSPT en tant que mécanisme explicatif de la revictimisation dans les relations amoureuses à l'adolescence. À l'aide d'analyses acheminatoires, les résultats de l'étude laissent entendre que le fait d'être victime d'agression sexuelle prédit davantage de violence subie dans les relations amoureuses. Les analyses de médiation révélant un effet indirect, indiquant que les victimes d'agression sexuelle présentent plus de SSPT et que ceux-ci, en retour, prédisent davantage de violence subie de la part d'un partenaire. Cet effet est observé à la fois pour la violence psychologique, physique et sexuelle subie au cours des 12 derniers mois. Ces observations sont cohérentes avec les résultats obtenus chez des populations adultes (Engstrom et al., 2008; Messing et al., 2012; Taft et al., 2005). Dans la seule étude précédente ayant considéré le type de violence subie, Messing et al. (2012) ont observé des associations significatives avec la violence psychologique et sexuelle. La contribution des SSPT à la revictimisation pourrait découler de leurs impacts sur la capacité de la victime à reconnaître les signes de danger et à émettre des comportements d'autoprotection face aux risques de subir de la violence (Arata, 2000; Chu, 1992; Orcutt et al., 2002; Risser et al., 2006). À ce titre, Risser et ses collègues (2006) semblent indiquer notamment que des niveaux d'hyperéveil chroniques pourraient en venir à diminuer la capacité à faire la discrimination entre fausses alarmes et signaux de danger réels, conduisant ainsi la personne à négliger certains signes de menaces.

En ce qui a trait aux différences liées au genre, la présente étude est l'une des premières à avoir étudié la relation entre l'agression sexuelle, les SSPT et la victimisation dans les relations amoureuses à la fois chez les filles et les garçons. Bien que les résultats bivariés indiquent que les filles rapportent davantage de SSPT et de violence psychologique et sexuelle, le genre ne modère pas significativement les relations entre l'agression sexuelle et les différentes formes de violence dans les modèles de médiation testés. Ces résultats semblent indiquer que le 
phénomène de la revictimisation dans les relations amoureuses est similaire chez les garçons et les filles. Cette observation est importante puisque les études québécoises portant sur le lien entre l'agression sexuelle et la victimisation dans les relations amoureuses des jeunes (Cyr et al., 2006; Hébert et al., 2008; Manseau et al., 2008; Tourigny et al., 2006) ont été conduites sur des échantillons exclusivement composés de filles. La présente étude laisse entendre que l'historique d'agression sexuelle est également un facteur de risque de revictimisation chez les garçons et que les SSPT constituent chez ces derniers un mécanisme de cette association au même titre que chez les filles.

Dans les analyses bivariées, les données de la présente étude révèlent que l'âge des participants est significativement associé à la violence psychologique, physique et sexuelle subie au sein des relations amoureuses, laissant entendre que les adolescents plus âgés rapportent vivre davantage de violence que les plus jeunes. Par contre, dans les analyses multivariées, l'âge apparaît influer seulement dans le modèle prédictif de la violence sexuelle subie. Ainsi, une relation curvilinéaire est établie, indiquant que la violence sexuelle pourrait d'abord diminuer et par la suite augmenter à partir de l'âge de 15 ans. Des études de modélisation tenant compte de la diversité des trajectoires ont illustré des patrons complexes des différentes trajectoires. Si pour certains jeunes, les taux de victimisation demeurent stables, pour d'autres, les risques de victimisation semblent augmenter avec le temps (Orpinas, Hsieh, Song, Holland et Nahapetyan, 2013). Pris dans l'ensemble, ces résultats soulignent l'importance de ne pas banaliser la violence subie dans les relations amoureuses puisque celle-ci ne semble pas constituer un phénomène transitoire et qu'elle est susceptible de s'accentuer, du moins à certaines périodes clés dans le développement à l'adolescence.

\section{Limites de l'étude et retombées pratiques}

La présente étude constitue un examen exploratoire du rôle des SSPT sur la revictimisation dans les relations amoureuses adolescentes et comporte certaines limites. D’abord, les analyses réalisées n'ont tenu compte ni de la sévérité ni du type d'agression sexuelle subie. Il faudrait de plus considérer d'autres formes de victimisation sexuelle, comme le harcèlement sexuel par les pairs, car il a été associé à la victimisation au sein du couple adolescent (Boivin, Lavoie, Hébert et Gagné, 2014). Dans le cadre des présentes analyses, les différentes dimensions (hyper- 
éveil, évitement, intrusion) des SSPT n'ont pas été considérées. De plus, la variance expliquée par les modèles demeure modeste, semblant indiquer que d'autres variables (p. ex., les stratégies de coping, le soutien reçu) peuvent interagir dans le lien entre l'agression sexuelle et la violence dans les relations amoureuses et devront faire l'objet d'études futures. Par ailleurs, les bêtas standardisés sont petits et ne pourraient être significatifs qu'en raison de la forte taille échantillonnale. De même, pour certains coefficients, les intervalles de confiance sont plutôt larges, ce qui laisse entendre que les résultats doivent être interprétés avec prudence. Finalement, le devis transversal de l'étude ne permet pas d'apprécier la temporalité des variables des SSPT et de violence dans les relations amoureuses afin de vérifier si l'apparition des SSPT précède l'expérience de violence. D’autres études ont par exemple examiné l'impact de la violence vécue de la part d'un partenaire sur le développement des SSPT (p. ex., Becker, Stuewig et McCloskey, 2010; Lilly, London et Bridgett, 2014; Matlow et DePrince, 2013). Les études futures devront s'appuyer sur un devis longitudinal afin de clarifier les relations de causalité entre les SSPT et la violence subie dans les relations amoureuses. Par ailleurs, les analyses futures seront à même d'évaluer la contribution spécifique des différentes dimensions (hyperéveil, évitement, intrusion) des SSPT dans la prédiction du risque de revictimisation. Une telle analyse pourrait offrir des cibles pertinentes pour les interventions auprès des adolescentes et adolescents victimes d'agression sexuelle en laissant entendre par exemple l'importance de traiter de façon prioritaire les symptômes d'hyperéveil.

Malgré les limites relevées, la présente étude contribue au corpus de connaissances sur les mécanismes liés à la revictimisation. En ayant recours à un vaste échantillon représentatif des adolescents et adolescentes des écoles secondaires du Québec, l'étude offre une première analyse de lien entre l'agression sexuelle, les SSPT et la revictimisation, et ce, tant pour les filles que pour les garçons. Le phénomène de la revictimisation à la suite de l'expérience d'agression sexuelle a jusqu'à maintenant été plus communément étudié chez des populations adultes. Pourtant, les développements scientifiques récents soulignent la nécessité de conceptualiser la revictimisation comme un cycle qui débute bien avant l'âge adulte et affecte les individus à divers stades du développement (Pittenger, Huit et Hansen, 2016). En ce sens, la présente étude constitue l'une des rares contributions à la compréhension du cycle de la revictimisation spécifiquement à la période de l'adolescence. 
Les données de l'étude présentent des implications importantes pour la prévention. D'une part, les données soulignent que la violence dans les relations amoureuses est malheureusement un phénomène qui peut accompagner les premières relations amoureuses de certains jeunes. Tant les adolescents que les adolescentes ayant vécu une agression sexuelle représentent une clientèle vulnérable, et des efforts particuliers doivent être entrepris pour réduire le risque de revictimisation. Les interventions visant les SSPT semblent une voie à privilégier. À l'aide de données provenant d'une étude clinique randomisée portant sur les effets de la cognitive processing therapy chez des femmes adultes victimes d'abus physique ou sexuel (Resick et al., 2008), Iverson et al. (2011) ont observé que les participantes ayant montré une diminution des SSPT et des symptômes dépressifs tout au long du traitement montraient également une réduction de la violence subie par un partenaire amoureux après un suivi de six mois. Ces résultats semblent indiquer qu'une attention particulière aux SSPT en tant que cible d'intervention centrale constitue une avenue prometteuse afin de réduire les risques de revictimisation chez les jeunes victimes d'agression sexuelle. Les études futures seront à même de vérifier cette possibilité.

\section{Références}

Arata, C. M. (2000). From Child Victim to Adult Victim: A Model for Predicting Sexual Revictimization. Child Maltreatment, 5(1), 28-38.

Banyard, V. L., Arnold, S. et Smith, J. (2000). Childhood sexual abuse and dating experiences of undergraduate women. Child Maltreatment, 5(1), 39-48.

Becker, K. D., Stuewig, J. et McCloskey, L. A. (2010). Traumatic stress symptoms of women exposed to different forms of childhood victimization and intimate partner violence. Journal of Interpersonal Violence, 25(9), 1699-1715.

Brooks-Russell, A., Foshee, V. et Ennett, S. (2013). Predictors of Latent Trajectory Classes of Physical Dating Violence Victimization. Journal of Youth et Adolescence, 42(4), 566-580.

Chu, J. A. (1992). The revictimization of adult women with histories of childhood abuse. Journal of Psychotherapy Practice et Research, 1(3), 259-269.

Coid, J., Petruckevitch, A., Feder, G., Chung, W.-S., Richardson, J. et Moorey, S. (2001). Relation between childhood sexual and physical abuse and risk of revictimisation in women: a cross-sectional survey. The Lancet, 358, 450-454.

Cyr, M., McDuff, P. et Wright, J. (2006). Prevalence and Predictors of Dating Violence Among Adolescent Female Victims of Child Sexual Abuse. Journal of Interpersonal Violence, 21(8), 1000-1017.

Daigneault, I., Hébert, M. et McDuff, P. (2009). Men's and women's childhood sexual abuse and victimization in adult partner relationships: A study of risk factors. Child Abuse et Neglect, 33(9), 638-647. 
Desai, S., Arias, I., Thompson, M. P. et Basile, K. C. (2002). Childhood victimization and subsequent adult revictimization assessed in a nationally representative sample of women and men. Violence and Victims, 17(6), 639-653.

DiLillo, D., Giuffre, D., Tremblay, G. C. et Peterson, L. (2001). A closer look at the nature of intimate partner violence reported by women with a history of child sexual abuse. Journal of Interpersonal Violence, 16(2), 116-132.

Engstrom, M., El-Bassel, N., Go, H. et Gilbert, L. (2008). Childhood Sexual Abuse and Intimate Partner Violence among Women in Methadone Treatment: A Direct or Mediated Relationship? Journal of Family Violence, 23(7), 605-617.

Finkelhor, D. et Browne, A. (1985). The traumatic impact of child sexual abuse: A conceptualization. American Journal of Orthopsychiatry, 55(4), 530-541.

Hébert, M., Brabant, M.-E. et Daigneault, I. (2013). Violence au sein des relations amoureuses et symptômes de stress post-traumatique chez les adolescentes ayant dévoilé une agression sexuelle. Journal international de victimologie, 11(2).

Hébert, M., Daigneault, I. et Van Camp, T. (2012). Agression sexuelle et risque de revictimisation à l'adolescence: Modèles conceptuels et défis liés à la prévention. Dans M. Hébert, M. Cyr et M. Tourigny (dir.), L'agression sexuelle envers les enfants. Tome II. (p. 171-223). Québec: Presses de l'Université du Québec.

Hébert, M., Lavoie, F., Vitaro, F., McDuff, P. et Tremblay, R. E. (2008). Association of child sexual abuse and dating victimization with mental health disorder in a sample of adolescent girls. Journal of Traumatic Stress, 21(2), 181-189.

Hébert, M., Tourigny, M., Cyr, M., McDuff, P. et Joly, J. (2009). Prevalence of childhood sexuel abuse and timing of disclosure in a representative sample of adults from Quebec. The Canadian Journal of Psychiatry, 54(9), 631-636.

Iverson, K. M., Gradus, J. L., Resick, P. A., Suvak, M. K., Smith, K. F. et Monson, C. M. (2011). Cognitive-behavioral therapy for PTSD and depression symptoms reduces risk for future intimate partner violence among interpersonal trauma survivors. Journal of Consulting and Clinical Psychology, 79(2), 193-202.

Kilpatrick, D. G., Ruggiero, K. J., Acierno, R., Saunders, B. E., Resnick, H. S. et Best, C. L. (2003). Violence and risk of PTSD, major depression, substance abuse/dependence, and comorbidity: Results from the National Survey of Adolescents. Journal of Consulting and Clinical Psychology, 71(4), 692-700.

Koss, M. P., Abbey, A., Campbell, R., Cook, S., Norris, J., Testa, M.,... White, J. (2007). Revising the SES: A collaborative process to improve assessment of sexual aggression and victimization. Psychology of Women Quarterly, 31(4), 357-370.

Koss, M. P. et Gidycz, C. A. (1985). Sexual Experiences Survey: Reliability and validity. Journal of Consulting and Clinical Psychology, 53(3), 422-423.

Koss, M. P., et Oros, C. J. (1982). Sexual Experiences Survey: A research instrument investigating sexual aggression. Journal of Consulting and Clinical Psychology, 50(3), 455-457.

Lilly, M. M., London, M. J., et Bridgett, D. J. (2014). Using SEM to examine emotion regulation and revictimization in predicting PTSD symptoms 
among childhood abuse survivors. Psychological Trauma: Theory, Research, Practice, and Policy, 6(6), 644-651.

Maikovich, A. K., Koenen, K. C. et Jaffee, S. R. (2009). Posttraumatic stress symptoms and trajectories in child sexual abuse victims: An analysis of sex differences using the National Survey of Child and Adolescent Well-Being. Journal of Abnormal Child Psychology, 37(5), 727-737.

Manseau, H., Fernet, M., Hébert, M., Collin-Vézina, D. et Blais, M. (2008). Risk factors for dating violence among teenage girls under child protective services. International Journal of Social Welfare, 17(3), 236-242.

Matlow, R. B. et DePrince, A. P. (2013). The influence of victimization history on PTSD symptom expression in women exposed to intimate partner violence. Psychological Trauma: Theory, Research, Practice, and Policy, 5(3), 241-250.

Messing, J. T., La Flair, L., Cavanaugh, C. E., Kanga, M. R. et Campbell, J. C. (2012). Testing Posttraumatic Stress as a Mediator of Childhood Trauma and Adult Intimate Partner Violence Victimization. Journal of Aggression, Maltreatment et Trauma, 21(7), 792-811.

Messman-Moore, T. L., Brown, A. L. et Koelsch, L. E. (2005). Posttraumatic symptoms and self-dysfunction as consequences and predictors of sexual revictimization. Journal of Traumatic Stress, 18(3), 253-261.

Nooner, K. B., Linares, L. O., Batinjane, J., Kramer, R. A., Silva, R. et Cloitre, M. (2012). Factors related to posttraumatic stress disorder in adolescence. Trauma, Violence, et Abuse, 13(3), 153-166.

Oddone Paolucci, E., Genuis, M. L. et Violato, C. (2001). A meta-analysis of the published research on the effects of child sexual abuse. The Journal of Psychology: Interdisciplinary and Applied, 135(1), 17-36.

Orcutt, H. K., Erickson, D. J. et Wolfe, J. (2002). A prospective analysis of trauma exposure: The mediating role of PTSD symptomatology. Journal of Traumatic Stress, 15(3), 259-266.

Pittenger, S. L., Huit, T. Z. et Hansen, D. J. (2016). Applying ecological systems theory to sexual revictimization of youth: A review with implications for research and practice. Aggression and Violent Behavior, 26, 35-45.

Rao, J. et Scott, A. (1981). The Analysis of Categorical Data from Complex Sample Surveys: Chi-Squared Tests for Goodness of Fit and Independence in Two-Way Tables. Journal of the American Statistical Association, 76(374).

Rao, J. et Scott, A. (1984). On Chi-Squared Tests for Multiway Contingency Tables with Cell Proportions Estimated from Survey Data on JSTOR. The Annals of Statistics, 12(1), 46-60.

Resick, P. A., Galovski, T. E., Uhlmansiek, M. O. B., Scher, C. D., Clum, G. A. et Young-Xu, Y. (2008). A randomized clinical trial to dismantle components of cognitive processing therapy for posttraumatic stress disorder in female victims of interpersonal violence. Journal of Consulting and Clinical Psychology, 76(2), 243-258.

Risser, H. J., Hetzel-Riggin, M. D., Thomsen, C. J. et McCanne, T. R. (2006). PTSD as a Mediator of Sexual Revictimization: The Role of Reexperiencing, Avoidance, and Arousal Symptoms. Journal of Traumatic Stress, 19(5), 687-698. 
Sandberg, D. A., Matorin, A. I. et Lynn, S. J. (1999). Dissociation, posttraumatic symptomatology, and sexual revictimization: A prospective examination of mediator and moderator effects. Journal of Traumatic Stress, 12(1), 127-138.

StataCorp. (2011). Stata Statistical Software: Release 12. College Station, TX: StataCorp LP.

Steinberg, A. M., Brymer, M. J., Decker, K. B. et Pynoos, R. S. (2004). The University of California at Los Angeles post-traumatic stress disorder reaction index. Current psychiatry reports, 6(2), 96-100.

Taft, C. T., Murphy, C. M., King, L. A., Dedeyn, J. M. et Musser, P. H. (2005). Posttraumatic Stress Disorder Symptomatology Among Partners of Men in Treatment for Relationship Abuse. Journal of Abnormal Psychology, 114(2), 259-268.

Tourigny, M., Lavoie, F., Vézina, J. et Pelletier, V. (2006). La violence subie par des adolescentes dans leurs fréquentations amoureuses: Incidence et facteurs associés. Revue de Psychoéducation, 35(2), 323-354.

Vézina, J. et Hébert, M. (2007). Risk Factors for Victimization in Romantic Relationships of Young Women: A Review of Empirical Studies and Implications for Prevention. Trauma, Violence, et Abuse, 8(1), 33-66.

Whitfield, C. L., Anda, R. F., Dube, S. R. et Felitti, V. J. (2003). Violent childhood experiences and the risk of intimate partner violence in adults: Assessment in a large health maintenance organization. Journal of Interpersonal Violence, 18(2), 166-185.

Wolfe, D. A., Scott, K., Reitzel-Jaffe, D., Wekerle, C., Grasley, C. et Straatman, A.-L. (2001). Development and validation of the conflict in adolescent dating relationships inventory. Psychological Assessment, 13(2), 277.

\title{
Sexual abuse and violence in dating relationships: The mediating role of post-traumatic stress symptoms
}

\begin{abstract}
This paper explores the mediating role of post-traumatic stress symptoms in the association between sexual abuse and different forms of victimization in dating relationships. The study is based on the Quebec Youth Romantic Relationships Survey, a survey that explores victimization experiences, including childhood sexual abuse, and any emotional, physical, and sexual dating violence in the past 12 months. The sample involved 8,194 students at 34 secondary schools in Quebec. Post-traumatic stress symptoms were evaluated by means of the UCLA PTSD Index. $15 \%$ of girls and $4 \%$ of boys reported a history of child sexual abuse. Path analyses suggest that sexual abuse is positively associated with post-traumatic stress symptoms, which in turn are associated with psychological, physical, and sexual victimization of dating partners. The study has significant implications for prevention of and intervention in dating violence involving victims of sexual abuse.
\end{abstract}

KEYWORDS - SeXual abuse, teen dating violence, post-traumatic stress, mediation, prevention. 


\section{Agresión sexual y violencia en las relaciones amorosas. El rol mediador del estrés post- traumático}

RESUMEN - El presente artículo busca documentar el rol de mediador de los sintomas de estrés post-traumático (SEPT) en la relación entre la agresión sexual y las diferentes formas de victimización vividas en el contexto de las relaciones amorosas. El estudio se basa sobre la Investigación sobre el recorrido amoroso de los jóvenes realizado en 34 escuelas secundarias de Quebec. Los jóvenes han completado un cuestionario sobre las experiencias de victimización, incluyendo la agresión sexual vivida durante la infancia y la violencia psicológica, física y sexual vivida en las relaciones amorosas de los últimos 12 meses. LOS SEPT han sido evaluados con la ayuda del índice UCLA PTSD. Sobre un total de 8194 adolescentes, 15\% de las mujeres y $4 \%$ de los varones han reportado haber vivido una agresión sexual. Los resultados de los análisis realizados sugieren que el hecho de haber sido víctima de una agresión sexual está asociada a un mayor grado de SEPT y que en contrapartida, los SEPT son positivamente asociados a la violencia psicológica, física y sexual ejercidas por una pareja. El presente estudio incluye las implicaciones prácticas para la prevención y la intervención en el ambito de la violencia en las relaciones amorosas de las víctimas de agresión sexual.

PALABRAS CLAVE - Agresión sexual, violencia en las relaciones amorosas, estrés posttraumático, mediación, prevención. 